\title{
The influence of footwear on foot motion during walking and running
}

\author{
Cédric Morio ${ }^{\mathrm{a}, \mathrm{b}, *}$, Mark J. Lake $^{\mathrm{c}}$, Nils Gueguen ${ }^{\mathrm{b}}$, Guillaume Rao ${ }^{\mathrm{b}}$, Laurent Baly ${ }^{\mathrm{a}}$ \\ a Oxylane Research, Department of Movement Sciences, 4 Boulevard de Mons BP 299, 59665 Villeneuve d'Ascq Cedex, France

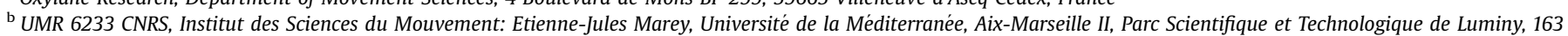 \\ Avenue de Luminy, 13288 Marseille Cedex 09, France \\ ${ }^{\mathrm{c}}$ Research Institute for Sport and Exercise Sciences, Liverpool John Moores University, Henry Cotton Campus, 15-21 Webster Street, Liverpool L3 2ET, UK
}

\section{A R T I C L E I N F O}

\section{Article history:}

Accepted 2 June 2009

\section{Keywords:}

Footwear

Walking

Running

Foot torsion

Forefoot width

\begin{abstract}
A B S T R A C T
There are evidences to suggest that wearing footwear constrains the natural barefoot motion during locomotion. Unlike prior studies that deduced foot motions from shoe sole displacement parameters, the aim of this study was to examine the effect of footwear motion on forefoot to rearfoot relative motion during walking and running. The use of a multi-segment foot model allowed accurate both shoe sole and foot motions (barefoot and shod) to be quantified. Two pairs of identical sandals with different midsole hardness were used. Ten healthy male subjects walked and ran in each of the shod condition.

The results showed that for barefoot locomotion there was more eversion of the forefoot and it occurred faster than for shod locomotion. In this later condition, the range of eversion was reduced by $20 \%$ and the rate of eversion in late stance by $60 \%$ in comparison to the barefoot condition. The sole constrained both the torsional (eversion/inversion) and adduction range of motion of the foot. Interestingly, during the push-off phase of barefoot locomotion the rate and direction of forefoot torsion varied between individuals. However, most subjects displayed a forefoot inversion direction of motion while shod. Therefore, this experiment showed that the shoes not only restricted the natural motion of the barefoot but also appeared to impose a specific foot motion pattern on individuals during the pushoff phase. These findings have implications for the matching of footwear design characteristics to individual natural foot function.
\end{abstract}

(c) 2009 Elsevier Ltd. All rights reserved.

\section{Introduction}

The motion of the forefoot relative to the rearfoot segment is usually described as plantarflexion/dorsiflexion (PF/DF), abduction/adduction (ABD/ADD), and eversion/inversion (EV/INV). Few studies have revealed a decrease in the amplitude of the natural motion of the forefoot relative to the rearfoot during shod locomotion (Freychat et al., 1996; Stacoff et al., 1989, 1991). Specifically, running shoes were found to decrease from $6-22^{\circ}$ to $0-6^{\circ}$ the $\mathrm{EV} / \mathrm{INV}$ amplitude relative to the natural barefoot torsion during running (Stacoff et al., 1989, 1991). They concluded that the stiffer the shoe, the more the natural motion of the foot was modified. This diminution in the EV/INV motion's amplitude was shown to be linked to an increase in rearfoot pronation, which has been recognized as a factor associated with lower-limb overuse injuries. Then, Freychat et al. (1996) found significant movement in forefoot to rearfoot ABD/ADD during barefoot running $\left(0.7 \pm 7.4^{\circ}\right)$ compared to the normal static position $\left(8.4 \pm 6.5^{\circ}\right)$.

\footnotetext{
* Correspondence to: Oxylane Research, Department of Movement Sciences, 4 Boulevard de Mons BP 299, 59665 Villeneuve d'Ascq Cedex, France. Tel.: +33320198336.

E-mail address: cedric.morio@oxylane-group.com (C. Morio).
}

They found different behaviour during running, "open foot" and "closed foot" that could be related to foot flexibilities. This relationship between foot flexibility and ABD/ADD range of motion (ROM) was confirmed later by Lee et al. (1999). This cadaveric study found $4.4^{\circ}$ of ADD ROM and $1.7^{\circ}$ of ABD ROM during simulated locomotion. Other studies looking at the effect of footwear on the forefoot EV/INV using different midsole hardness (Nigg and Bahlsen, 1988), spikes and normal running shoes (Stacoff et al., 1991) or different sport shoes (Stacoff et al., 1996) However, Stacoff et al. did not use systematic modifications in footwear sole properties to investigate foot motion resulting from constraints. Moreover, all the previous studies looked at forefoot EV/INV only with 2D high speed video.

Except Lee et al. (1999), the preceding studies focused on 2D kinematics of the forefoot to rearfoot motion in the frontal (Nigg and Bahlsen, 1988; Stacoff et al., 1989, 1991, 1996) or transverse planes (Freychat et al., 1996). In recent years, 3D multi-segment models of the foot have been used to study foot motions while barefoot walking (Carson et al., 2001; Hunt et al., 2001; Kidder et al., 1996; Leardini et al., 1999; Rattanaprasert et al., 1999) and running (Pohl and Buckley, 2008; Pohl et al., 2006, 2007) and during shod running (Eslami et al., 2007; Stacoff et al., 2000, 2001; Wolf et al., 2008). Using 2 segments foot models, Pohl et al. (2007) demonstrated better coupling pattern during running than 
walking between rearfoot $\mathrm{EV} / \mathrm{INV}$ and shank rotation and between rearfoot $\mathrm{EV} / \mathrm{INV}$ cross forefoot $\mathrm{PF} / \mathrm{DF}$. They also found significantly greater forefoot to rearfoot $\mathrm{DF}$ and $\mathrm{ABD}$ excursions during running than walking. No significant difference between barefoot and shod running for the rearfoot to tibia eversion was found (Eslami et al., 2007; Stacoff et al., 2000, 2001). However, Wolf et al. (2008) found significant footwear constrain of the natural foot movement in children, especially in forefoot width and forefoot to rearfoot $\mathrm{EV} / \mathrm{INV}$ during the walking stance phase. Thus, questions remain about the influence of the shoe design parameters on the natural foot movement in adults during running. This can be partly explained by the difficulty of monitoring foot movement inside a shoe. Some solutions involved cutting windows in the shoe upper in order to see skin markers (Stacoff et al., 1996; Wolf et al., 2008) or more directly by using sandals (Eslami et al., 2007).

To our knowledge there was no previous study which looked at the sole motion as a parameter to discuss the changes in foot motion during shod and barefoot, walking and running. Hence, a preliminary study was done to investigate the foot and sole movements during walking and running (3 subjects, same methodology as in the present study). Twelve biomechanically relevant variables were extracted from this pilot work to describe the foot and sole movements during both walking and running.

The first objective of the present investigation was to show how the forefoot to rearfoot relative movement was influenced and restricted by footwear. It was hypothesized that the shoe constrains natural foot movements in ABD/ADD and EV/INV. The second objective examined the effect of gait mode, walking or running, on this restriction of foot movement. It was hypothesized that during running the foot might apply more force on the footwear than during walking helping to counteract any restriction. Therefore, a two-segment foot model was used to look at the influence of footwear on those motions in the two different gait modes using two different sandals with different midsole hardness.

\section{Materials and methods}

\subsection{Subject population}

Ten healthy, male subjects (age: $25.4+6.4$ years, height: $1.78+0.05 \mathrm{~m}$, weight: $81.4 \pm 13.9 \mathrm{~kg}$ ) volunteered to participate in this study. They were free from lowerlimb injury and wore a shoe size of 43.5 (EU). All the subjects were rearfoot strikers.

\subsection{Experimental protocol}

After practise trials to control the speed and the right foot landing area, the subjects were asked to perform at least 10 trials for each condition along a $15 \mathrm{~m}$ pathway. Three different conditions for walking and running were compared in this study, a barefoot condition and two different shod conditions. For the shod conditions, identical sandals (same construction and size, inner length $275 \mathrm{~mm}$ and inner width $95 \mathrm{~mm}$ ) with two different midsole hardness were used (soft: Asker 40C and hard: Asker 65C). This hardness corresponded to the extreme limits reachable using the same EVA foam process. Sandals were chosen because they allowed the same skin markers to be used for assessing foot movement during barefoot and shod locomotion. The sandals were carefully tightened by the experimenter, once tightened the straps were also secured by tapping to prevent loosening. Gait speed was monitored using photocell timing gates before and after the data capture area where the speed was assumed to be constant. Subjects ran at $4.20+0.08 \mathrm{~m} \mathrm{~s}^{-1}$ and walked at $1.71 \pm 0.03 \mathrm{~m} \mathrm{~s}^{-1}$.

The sandals stiffness were measured by a hydraulic testing device (Zwick/ Roell, Germany) previously used by Schwanitz and Odenwald (2008). The mechanical test consisted in a sinus compression of the sandal midsole under the heel part between $100 \mathrm{~N}$ (preload) and four different maximum loads 400,800 , 1200 and $1600 \mathrm{~N}$. The force-deformation data were collected and the stiffness was calculated as the force variation (i.e., 300, 700, 1100 and $1500 \mathrm{~N}$ ) divided by the corresponding displacement of the midsole.

Kinematic and kinetic data were captured on the right foot and sole only. The kinematic data were collected at $500 \mathrm{~Hz}$ by $9 \operatorname{ProReflex}^{\mathbb{B}}$ MCU1000 cameras
(Qualisys, Sweden) and synchronized with ground reaction force data (Kistler Switzerland) collected at $2000 \mathrm{~Hz}$.

Seventeen reflective markers were used to define and track the right foot and sole segments (Fig. 1a). Similar to Pohl et al. (2006, 2007), a two-segment foot model was used to characterise dynamic foot motion, with the rearfoot defined as the calcaneus (Fig. 1b) and the forefoot as the five metatarsals (Fig. 1c). A comparable two-segment model of the sole was also used to define the sole motion (Fig. 1d). The same axis system was used for both models in order to compare sole and foot motions.

\subsection{Data analysis}

The stance phase (heel strike to toe-off) was determined using the force platform data ( $20 \mathrm{~N}$ threshold). As the gait speed was consistent between trials and subjects, all the stance phases were normalized from $0 \%$ to $100 \%$.

The kinematic raw data were filtered with a fourth order low-pass bidirectional Butterworth filter at $30 \mathrm{~Hz}$ for running and $15 \mathrm{~Hz}$ for walking. Cut-off frequencies were set in order to keep $95 \%$ of the raw signal frequency content. The forefoot to rearfoot relative motions were calculated in accordance with the recommendations of the International Society of Biomechanics for the definition of

a

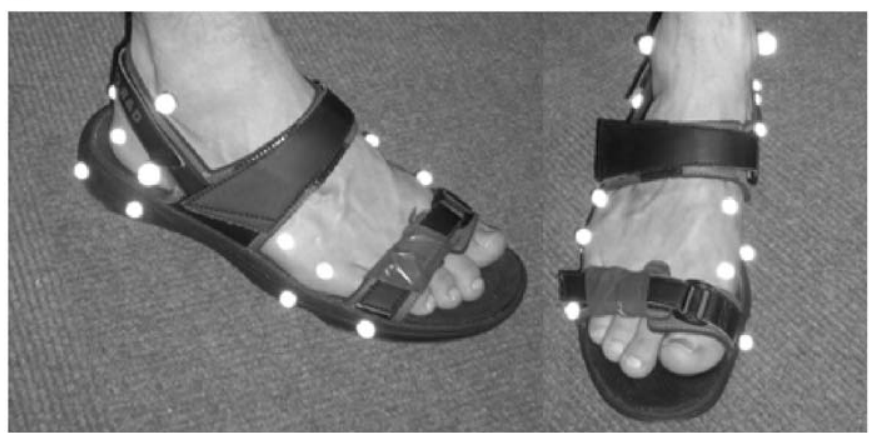

b



C

MB1

d

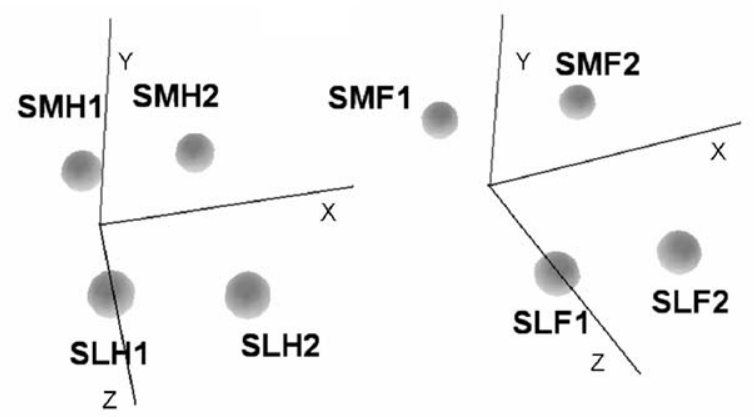

Fig. 1. The picture (a) presents the marker placement on the foot and the sandal's sole. This figure represents the 2 segments foot model and the 2 segments sole model. The rearfoot segment (b) is defined by the markers FLH1, FHL2, FMH1 and FMH2 and the tracking markers are FHL1, FMH1 and Heel. The forefoot segment (c) is defined by the bases and the heads of the first and the fifth metatarsals MB1, MB5, MH1 and MH5 and the four markers are used as tracking markers. The sole model (d) is define as a 2 segments model with a rearsole and a foresole, the rearsole is defined by SLH1, SLH2, SMH1 and SMH2 with only SLH2, SMH1 and SMH2 as tracking markers (the SLH1 has not been used for tracking because of the compression of that part of the sole in the first times of the landing) and the foresole is defined by SLF1, SLF2, SMF1 and SMF2 with the four as tracking markers. 
the orientation of the joint co-ordinate system (Wu et al., 2002) using a Cardan sequence of $Z(P F / D F), X(E V / I N V), Y(A B D / A D D)$. Separate calibrations were performed for the foot and sandals. The calibration for the foot segments was used to set the zero reference position in every condition (i.e., walking, running, barefoot and shod). The zero reference position for the foot segments was obtained during a barefoot two-legged standing trial. For the sandal soles, two calibrations were performed, one for each type of sole (soft and hard). For each sole, the reference position was obtained while standing in the given sandal and was subsequently used as the sole segment references.

The rearfoot, forefoot, rearsole and foresole segments were modelled using Visual3D ${ }^{\mathbb{R}}$ software (C-Motion, USA). The angular motions of the forefoot relative to the rearfoot and of the foresole relative to the rearsole were also calculated with Visual3D, and then Matlab ${ }^{\mathbb{R}} 7$ software (The Mathworks, USA) was used to calculate key variables from those datasets.

\subsection{Definition of key variables}

The forefoot to rearfoot relative motion was very consistent for each subject. An example of this low intra-subject variability is illustrated in Fig. 2. Consequently, the data analysis focused on the mean curves for each subject.

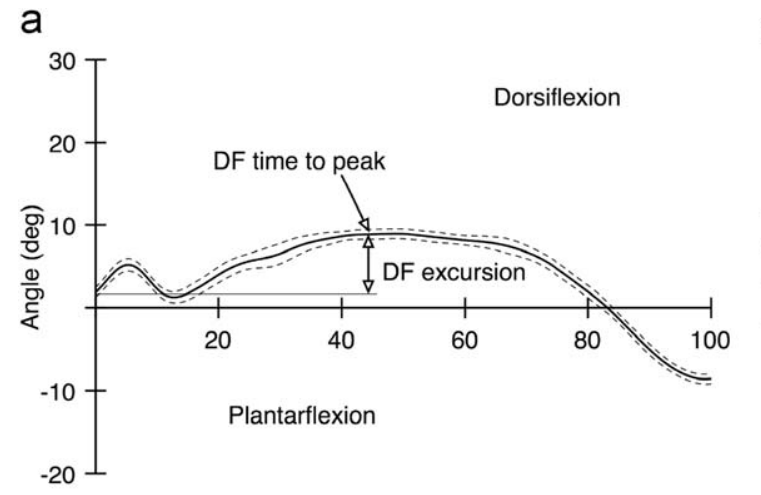

b

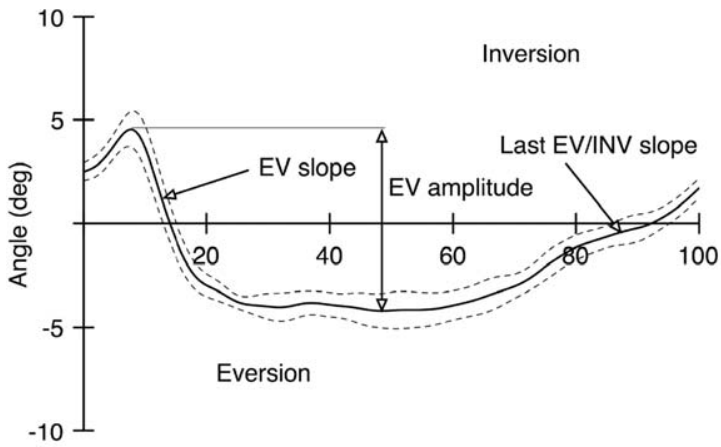

C



Stance duration (\%)
Twelve discrete key variables were chosen to describe foot (8 variables) and sole ( 4 variables) motion (Fig. 2). Four foot motion variables were the same as those used by Pohl et al. (2006), the forefoot to rearfoot DF and ABD excursions and time to peak in percentage of the stance phase. The DF and ABD excursions were defined as the difference between the maximum value and the value at foot strike. The other 8 variables were extracted from the pilot study to further explore potential differences between barefoot and shod conditions. The ABD/ADD peak to peak amplitude (ADD amplitude) was included. Forefoot to rearfoot EV/INV was described by the first EV slope from the first INV peak to $10 \%$ of the stance phase later, then by the EV/INV peak to peak amplitude (EV amplitude), and finally the maximum slope of the EV/INV curve in the last $25 \%$ of stance. Sole flexion was characterised by the amplitudes of the two flexion peaks and sole torsion amplitude as EV/INV peak to peak amplitude and the maximum INV slope during the first $30 \%$ of stance.

Two other variables were calculated to examine the forefoot width expansion caused by the foot loading during the stance phase and its restriction by the sandals. This "forefoot spreading" was calculated as the percentage of variation of the metatarsal bases $(\Delta$ Bases $=$ distance $\mathrm{MB} 1-\mathrm{MB} 5)$ and the metatarsal heads $(\Delta$ Heads $=$ distance $M H 1-M H 5)$. To explore the gait mode effect, forefoot spreading variables were compared from walking and running to normal standing respectively. Then, to explore the shod condition effect, the forefoot spreading variables were compared only for normal standing.

d
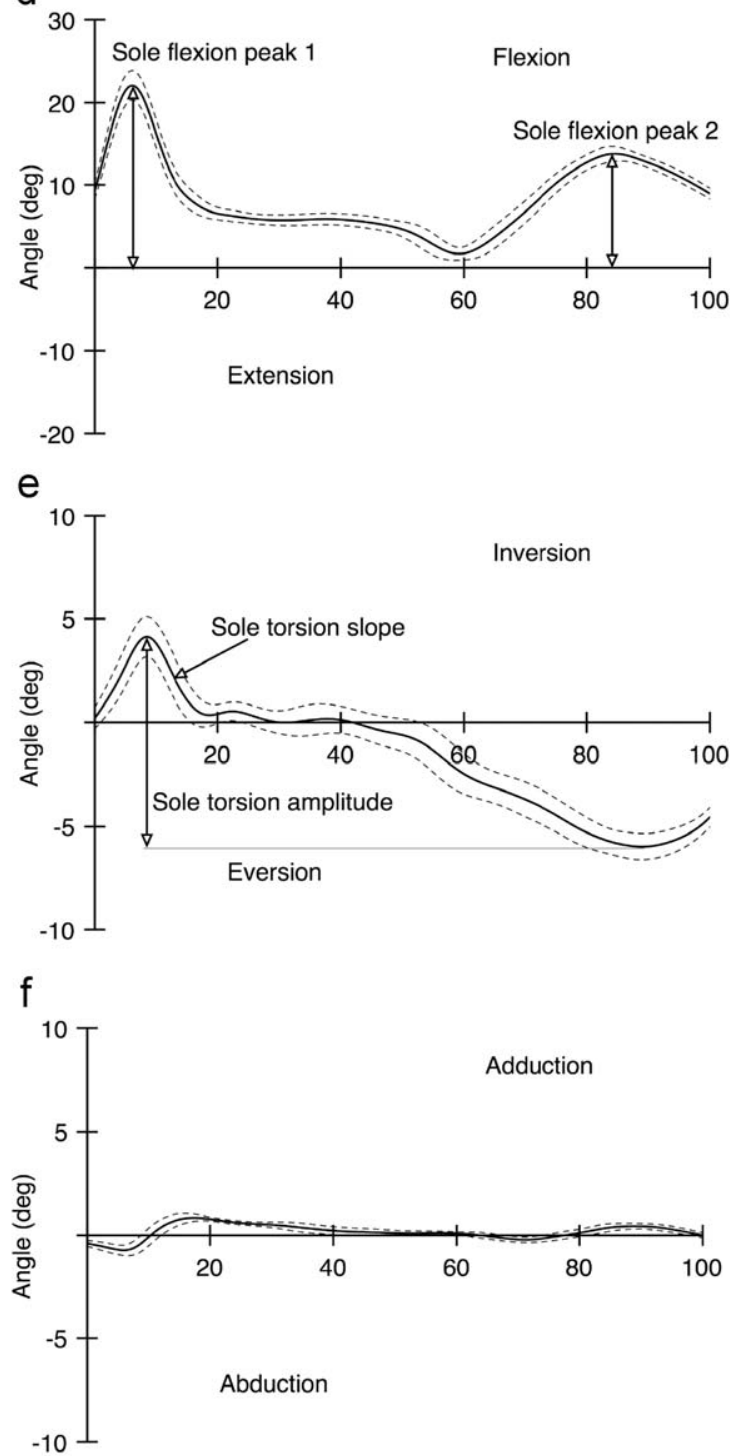

Stance duration (\%)



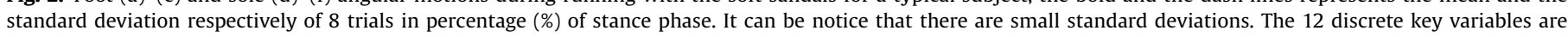

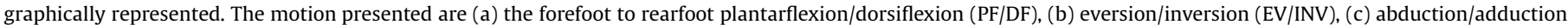
(ABD/ADD) and, (d) the foresole to rearsole flexion, (e) torsion or eversion/inversion and (f) abduction/adduction. 

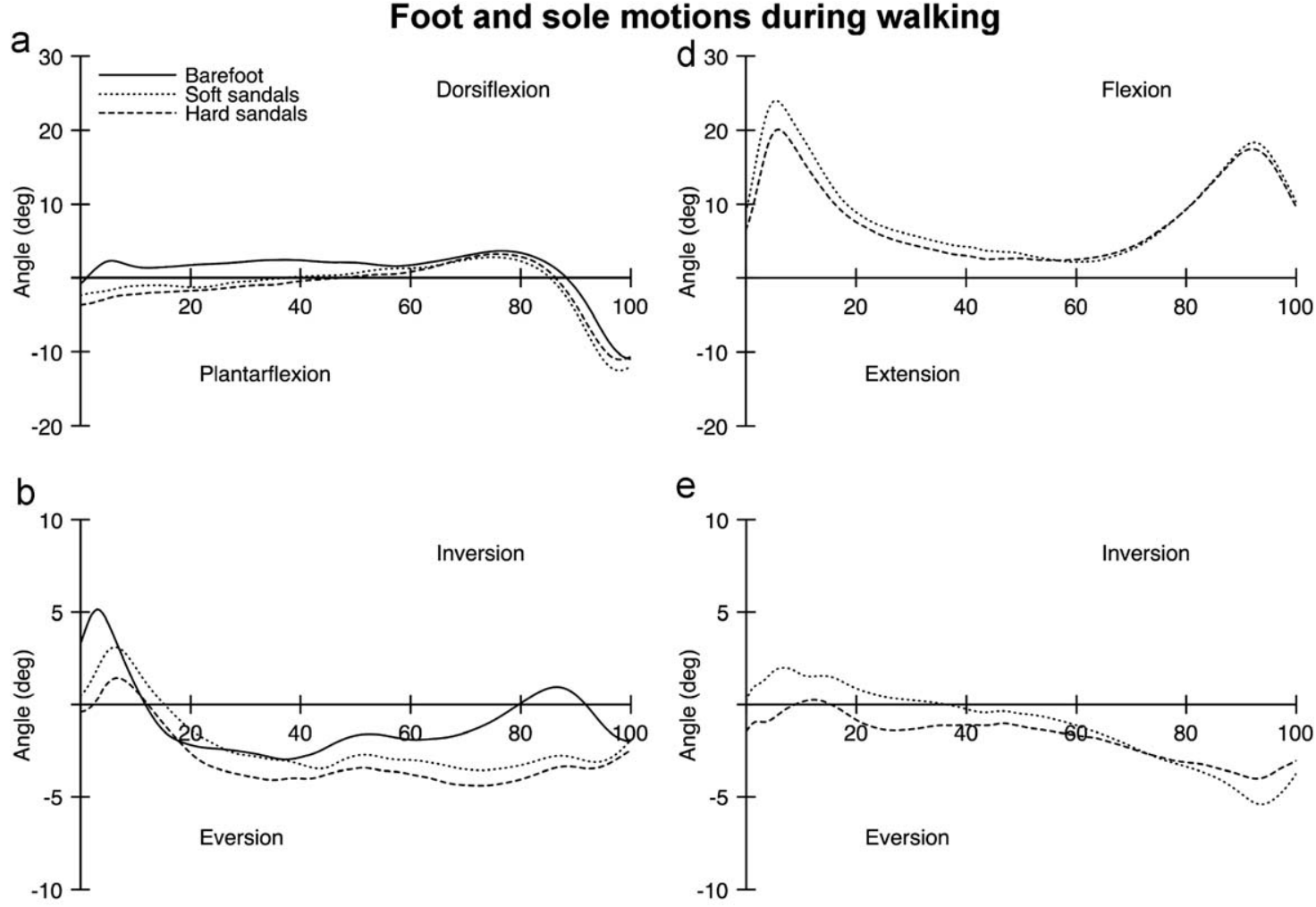

e

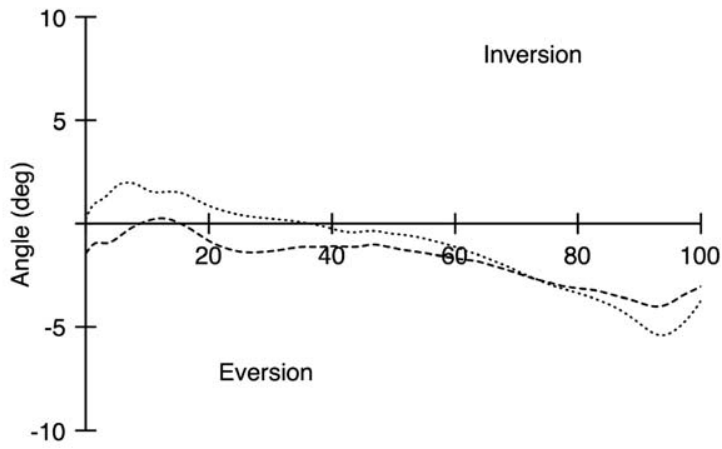



Stance duration (\%) $\mathrm{f}$

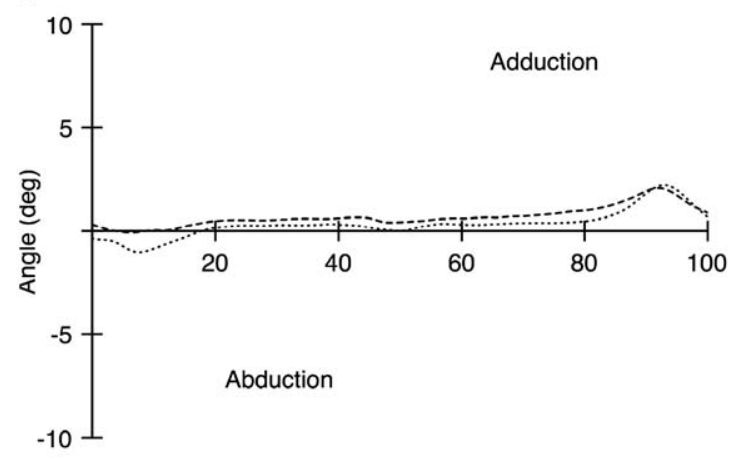

Stance duration (\%)

Fig. 3. Foot (a)-(c) and sole (d)-(f) motions during walking in the sagittal (a) (d), frontal (b) (e) and horizontal (c) (f) planes. All the curves are mean over all the subjects and they are presented in percentage of the stance phase, barefoot (plain), soft sandals (dot) and hard sandals (dash). The motions presented are (a) the forefoot to rearfoot plantarflexion/dorsiflexion (PF/DF), (b) eversion/inversion (EV/INV), (c) abduction/adduction (ABD/ADD) and (d) the foresole to rearsole flexion, (e) torsion or eversion/ inversion and (f) abduction/adduction.

\subsection{Statistics}

Foot variables were analysed using a two-way analysis of variance (ANOVA) for two gait modes and three shod conditions. Forefoot spreading variables were analysed with a one-way ANOVA for both gait mode and shod condition effect. Sole variables were analysed with a two-way ANOVA for two gait modes and two hardness conditions. Then, Tukey's multiple comparison post-hoc tests $(p<0.05)$ was used to assess differences between conditions.

\section{Results}

\subsection{Sandals stiffness}

The measured stiffness of the soft sandals for the four different loads $(400,800,1200$ and $1600 \mathrm{~N})$ were $99,129,199$ and $267 \mathrm{~N} /$ $\mathrm{mm}$, respectively, and the measured stiffness of the hard sandals were $170,183,227$ and $296 \mathrm{~N} / \mathrm{mm}$, respectively.

\subsection{Foot variables}

Patterns of foot motion during walking and running were broadly similar between barefoot and shod conditions but some significant differences could be observed. As shown in Figs. 3 and 4, clear differences were observed in EV/INV and last $20 \%$ of ABD/ADD.

ANOVAs showed a significant main effect for the shod conditions on several foot motion variables (Table 1). Thus, EV amplitude showed greater values during barefoot locomotion. The EV slope and EV/INV max slope showed significant differences during barefoot compared to shod conditions. However, subjects presented different EV/INV pattern during the barefoot push-off. Indeed, the variability was reduced during shod gait, with 9 of 10 subjects displaying an inversion pattern during shod push-off, whereas during barefoot locomotion there were more distinct pattern, EV for 4 subjects, INV for 5 subjects and neutral pattern 

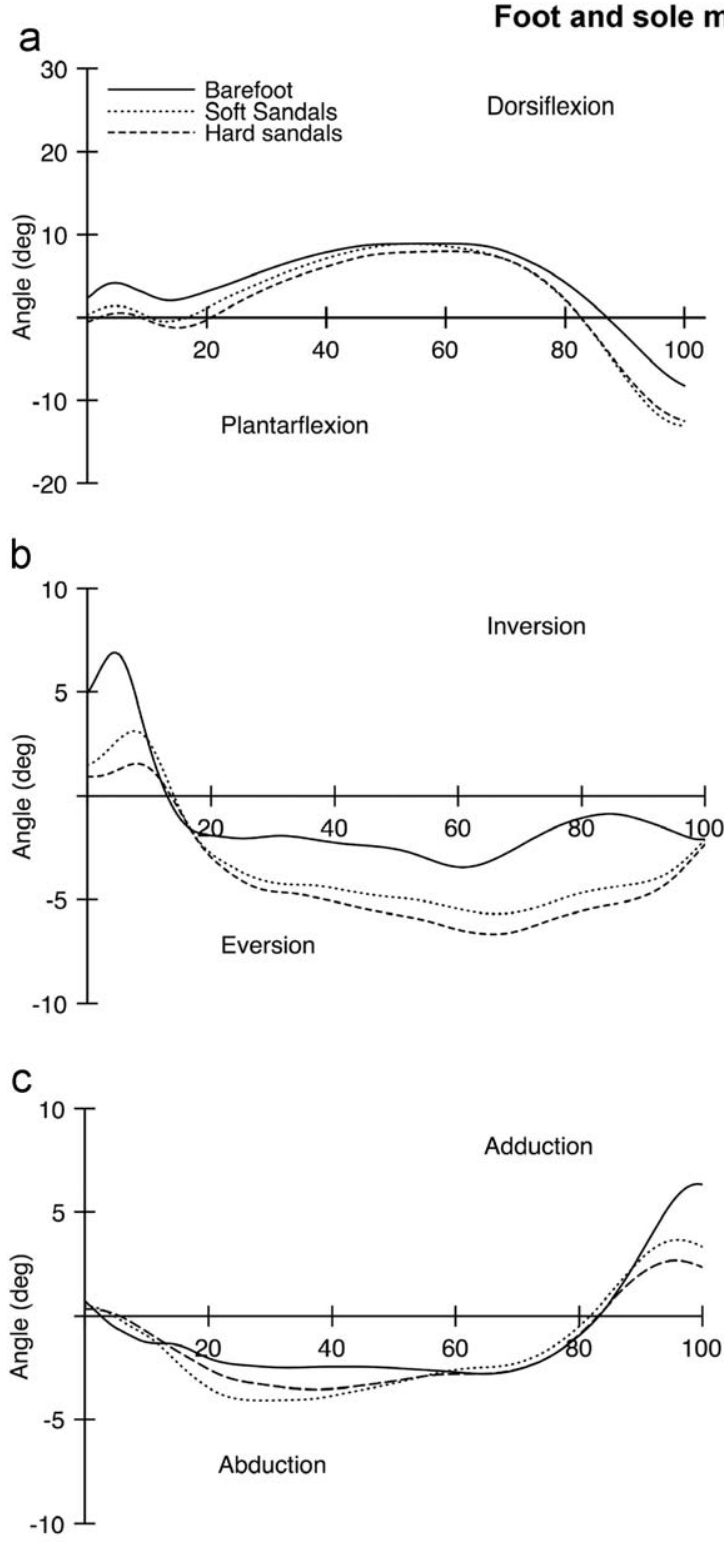

Stance duration (\%)

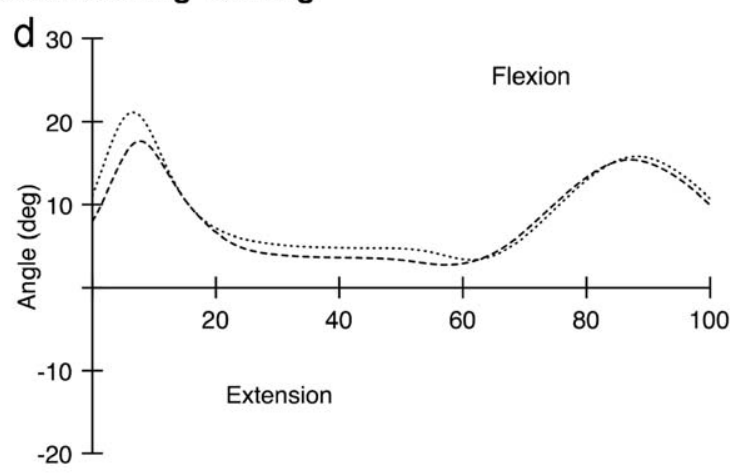

e

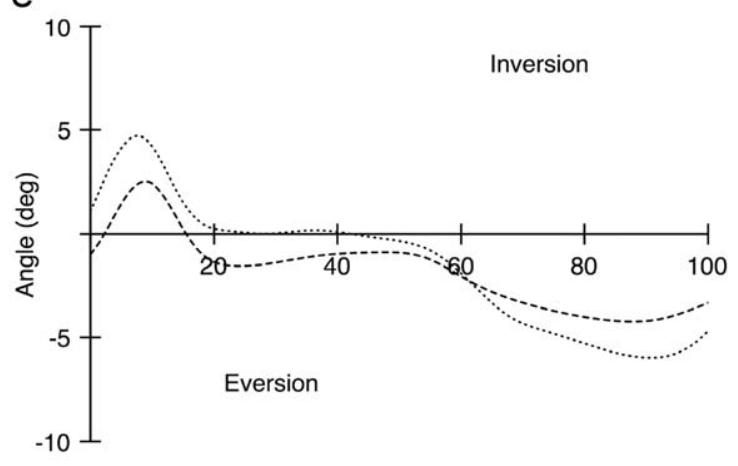

f

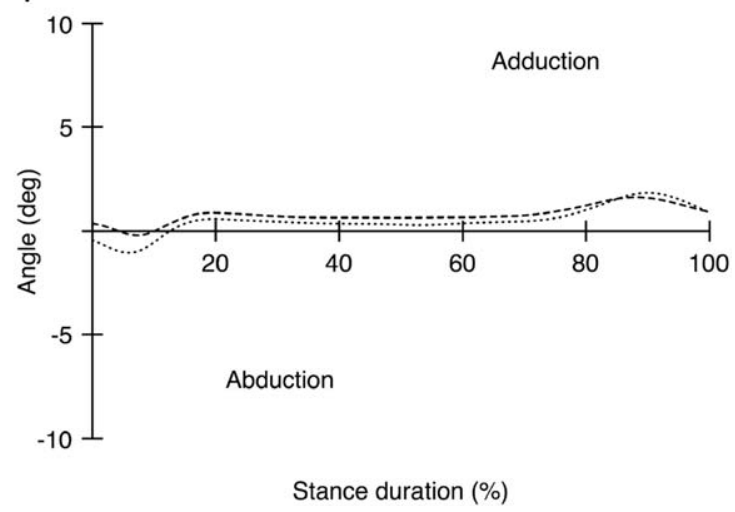



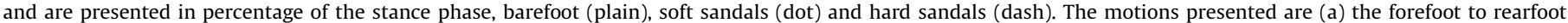

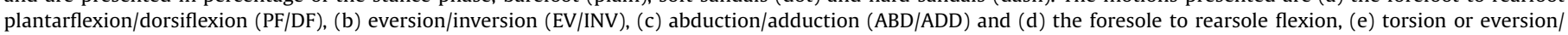
inversion and (f) abduction/adduction.

for 1 subject (Fig. 5). ABD excursion only presented differences between the barefoot and the soft sandals during running. ADD amplitude was significantly higher during barefoot than with both shod conditions. During walking, ADD amplitude was also significantly higher in the soft relative to the hard sandals. During walking, DF excursion was smaller in the barefoot condition relative to the both shod conditions. Then, a significant main effect was seen for the gait mode on the forefoot to rearfoot discrete variables. DF, ABD excursions and EV amplitude were greater during running than during walking. In contrast, ADD amplitude presented slightly smaller amplitude during running. Finally, an interaction between gait modes and shod conditions was observed for the EV slope variable. This was explained by larger increase in EV slope going from shod walking to barefoot running. The soft sandal during running also demonstrated faster early EV compared to walking with the hard sandal.
A gait mode effect was demonstrated for both forefoot spreading variables, $\Delta$ Bases and $\Delta$ Heads (Table 2 ). The spreading was greater during running compared to walking trials for each condition. Then, a shod condition effect was studied during standing trials. Both variables showed that the hard sandals constrained the natural spreading for the $\Delta$ Bases and $\Delta$ Heads while the soft sandals constrained the metatarsals heads only.

\subsection{Sole variables}

Sole torsion patterns were roughly similar to foot patterns only for the first $20 \%$ of the EV/INV component. Unlike the forefoot to rearfoot PF/DF curves which displayed one big hump (Figs. 3a and $4 a)$, the sole flexion showed three phases. These three phases were a first flexion from $0 \%$ to $20 \%$ of the stance phase, a sole flat 
Table 1

The mean (straight) and standard deviation (italic) of the 8 discrete key variables concerning the foot motion for all 10 subjects.

\begin{tabular}{|c|c|c|c|c|c|c|}
\hline & \multicolumn{3}{|l|}{ Walk } & \multicolumn{3}{|l|}{ Run } \\
\hline & Barefoot & Soft & Hard & Barefoot & Soft & Hard \\
\hline \multirow[t]{2}{*}{ DF excursion (deg.) ${ }^{\mathrm{G}, \mathrm{S}}$} & 4.8 & 5.5 & 6.8 & 6.9 & 8.7 & 9.1 \\
\hline & 2.1 & 1.5 & 2.3 & 2.4 & 2.1 & 2.2 \\
\hline \multirow{2}{*}{$\begin{array}{l}\text { DF time to peak (\%stance) } \\
\mathrm{S}\end{array}$} & 73.3 & 70.0 & 75.4 & 56.8 & 54.1 & 59.5 \\
\hline & 8.9 & 10.7 & 6.0 & 7.8 & 5.2 & 7.3 \\
\hline \multirow[t]{2}{*}{ ABD excursion (deg.) ${ }^{\mathrm{G}, \mathrm{S}}$} & -2.6 & -3.1 & -2.5 & -3.4 & -4.5 & -4.1 \\
\hline & 2.0 & 1.7 & 1.8 & 2.2 & 2.2 & 2.5 \\
\hline \multirow{4}{*}{$\begin{array}{l}\text { ABD time to peak } \\
{\text { (\%stance })^{\mathrm{G}, \mathrm{I}}}^{\text {ADD amplitude (deg. })^{\mathrm{G}, \mathrm{S}}}\end{array}$} & 19.4 & 26.0 & 29.9 & 45.6 & 35.8 & 42.9 \\
\hline & 20.4 & 17.3 & 21.1 & 22.3 & 10.8 & 14.2 \\
\hline & 11.5 & 9.8 & 8.3 & 9.6 & 8.2 & 6.9 \\
\hline & 1.8 & 2.0 & 1.6 & 2.6 & 2.8 & 2.6 \\
\hline \multirow[t]{2}{*}{ EV slope $\left(\text { deg. } s^{-1}\right)^{G, S, ~ I ~}$} & -98.1 & -52.8 & -44.0 & -371.9 & -232.3 & -142.8 \\
\hline & 32.7 & 28.2 & 27.4 & 98.5 & 126.9 & 138.4 \\
\hline \multirow[t]{2}{*}{ EV amplitude (deg.) ${ }^{\mathrm{G}, \mathrm{S}}$} & 9.5 & 8.2 & 7.9 & 12.0 & 10.7 & 10.0 \\
\hline & 2.9 & 2.8 & 2.7 & 3.9 & 4.6 & 4.9 \\
\hline EV/INV max slope & -67.5 & 43.7 & 39.4 & -10.9 & 116.8 & 136.0 \\
\hline$\left(\operatorname{deg} . s^{-1}\right)^{S}$ & 60.3 & 43.1 & 37.7 & 179.0 & 115.9 & 114.8 \\
\hline
\end{tabular}

A $(G)$ indicates a main effect of the gait mode, a (S) a main effect of the shod conditions and $(\mathrm{I})$ represents a significant $(p<0.05)$ interaction between the gait mode and the shod conditions.



Fig. 5. The upper chart represents the EV/INV max slope of the 10 subjects during the last $25 \%$ of the stance phase for the 3 shod conditions during running. For barefoot running 5 subjects show a last inversion of the forefoot relative to the rearfoot (positive value), 1 shows a neutral pattern and 4 show an eversion of the forefoot (negative values). For shod running, 9 subjects show a last inversion against 1 still in eversion, but with a trend for diminution of that last inversion pattern.

phase from $20 \%$ to $70 \%$, and a second flexion from $70 \%$ to $100 \%$. Then, the sole did not present ABD/ADD component. The two flexion peaks were influenced by the gait mode but only the first by the midsole hardness (Table 3 ). Both sole torsion variables presented a gait mode effect and a hardness effect. The sole torsion amplitude was greater for the soft sandals during running. In contrast, the sole torsion slope presented similar trends with an interaction, where running mode reduced the shod condition effect compared to walking.

\section{Discussion}

The first objective of this study was to highlight the footwear influence on the restriction of foot motion. The corresponding hypothesis was that the footwear constraints the natural foot motion. Our results presented a restricted motion of the foot in
Table 2

Mean (straight) and standard deviation (italic) of the forefoot spreading in two different locations: metatarsals bases ( $\Delta$ Bases) and metatarsals heads ( $\Delta$ Heads).

\begin{tabular}{llcclcc}
\hline & $\begin{array}{l}\text { Walk } \\
\text { Barefoot }\end{array}$ & Soft & Hard & $\begin{array}{l}\text { Run } \\
\text { Barefoot }\end{array}$ & Soft & Hard \\
\hline BBases (\%) $^{\mathrm{G}}$ & 0.8 & 1.1 & 1.7 & 2.3 & 2.3 & 3.1 \\
& 1.0 & 1.1 & 1.4 & 0.9 & 1.7 & 1.7 \\
HHeads (\%) $^{\mathrm{G}}$ & 1.1 & 0.9 & 1.6 & 2.9 & 2.2 & 2.9 \\
& 0.8 & 1.3 & 1.7 & 1.2 & 1.8 & 1.4 \\
& Standing calibration & & & & \\
SBases (\%) & $0.0^{\mathrm{H}}$ & $-0.7^{\mathrm{H}}$ & $-2.4^{\mathrm{B}, \mathrm{S}}$ & & & \\
& - & 0.9 & 1.4 & & & \\
SHeads (\%) & $0.0^{\mathrm{S}, \mathrm{H}}$ & $-2.5^{\mathrm{B}}$ & $-3.5^{\mathrm{B}}$ & & & \\
& - & 1.8 & 1.8 & & & \\
\hline
\end{tabular}

The top table shows the forefoot spreading value for each condition relative to their respective standing calibration. There is a significant effect of the gait mode (G) but no significant difference within gait mode. The shod condition effect is presented in the bottom table, where " $\mathrm{B}$ " notifies a significant difference with the barefoot condition, " $\mathrm{S}$ " with the soft sandals and " $\mathrm{H}$ " with the hard sandals.

Table 3

The mean (straight) and standard deviation (italic) of the 4 discrete key variables concerning the sole motion for all 10 subjects.

\begin{tabular}{|c|c|c|c|c|}
\hline & $\begin{array}{l}\text { Walk } \\
\text { Soft }\end{array}$ & Hard & $\begin{array}{l}\text { Run } \\
\text { Soft }\end{array}$ & Hard \\
\hline \multirow[t]{2}{*}{ Sole flexion peak 1 (deg.) $)^{\mathrm{G}, \mathrm{S}}$} & 24.2 & 20.2 & 21.7 & 18.2 \\
\hline & 2.5 & 3.3 & 3.4 & 3.2 \\
\hline \multirow[t]{2}{*}{ Sole flexion peak 2 (deg.) ${ }^{\mathrm{G}, \mathrm{I}}$} & 18.8 & 17.7 & 16.0 & 15.5 \\
\hline & 3.7 & 3.2 & 3.0 & 2.6 \\
\hline \multirow[t]{2}{*}{ Sole torsion slope $\left(\text { deg. } s^{-1}\right)^{\mathrm{G}, ~ S, ~ I ~}$} & -156.2 & -343.7 & -382.3 & -403.9 \\
\hline & 155.4 & 179.5 & 118.1 & 139.1 \\
\hline \multirow[t]{2}{*}{ Sole torsion amplitude (deg.) $)^{\mathrm{G}, \mathrm{S}}$} & 8.4 & 5.7 & 11.5 & 7.8 \\
\hline & 1.7 & 1.6 & 2.7 & 2.2 \\
\hline
\end{tabular}

A $(G)$ indicates a main effect of the gait mode, a $(S)$ a main effect of the shod conditions and a (I) represents a significant $(p<0.05)$ interaction between the gait mode and the shod conditions.

the frontal and horizontal planes, but not in the sagittal plane. Indeed, this was confirmed for EV/INV and ABD/ADD foot motions but not for PF/DF motion. Several foot key variables showed that the sandals constrained the natural foot motion, ADD amplitude, EV slope, EV amplitude, $\Delta$ Bases and $\Delta$ Heads. EV/INV max slope variability also supported that notion. Identical results were found in children when comparing barefoot and shod gait (Wolf et al., 2008). These authors found that the footwear constrained foot torsion, forefoot spreading, and foot pronation during push-off. The present study presented the same differences in push-off patterns; this was shown by the EV/INV variability. Additionally, the sandals did not display any ABD/ADD motion. It could be hypothesized that the sandals did not follow the natural foot motion and therefore constrained this motion. Nevertheless, as proposed by Wolf et al. (2008) the forefoot to rearfoot ABD/ADD could be also dependent on the flexibility of the upper that was not considered in the present study. Regarding our results on the sole motion, we can assume that it was the sandal that constrained the foot motion. Indeed, the sole torsion amplitude was significantly diminished in the hard compared to the soft sandals. Concurrently, the forefoot to rearfoot EV amplitude was reduced when wearing hard sandals compared to soft one.

In contrast, some variables did not show any significant constrain of the sandals, DF and ABD excursions, $\Delta$ Heads, second sole flexion peak. For example, the first sole flexion peak demonstrated a smaller amount of motion with the hard sandals compare with the soft ones, although DF excursion present an increased with hard sandals compared to soft ones. Oleson et al. 
(2005) suggest that as the foot stiffness was significantly greater than the sole stiffness, it was the foot bending which governed shoe/foot metatarsals DF during running. Our result cannot confirm this but it clearly shows that there was at least no restriction of the forefoot DF excursion due to the sandals. Although midsole hardness influence was reported previously (Hamill et al., 1992; Hardin et al., 2004; Nigg and Bahlsen, 1988), the present study found similar foot motion between both shod conditions. So a systematic change in the midsole hardness either did not affect the amount of footwear constrain or was too small compared to the inter-individual variability (Stacoff et al., 2001). Although there was clear difference of stiffness property of the sandals, no influence on foot motion was demonstrated in both gait modes. The hard sandals were stiffer than the soft ones for any load conditions. The mechanical testing also showed a reduction of this difference between the stiffness of the soft and the hard sandals when the solicitation load increased. The similar foot movement pattern between soft and hard sandal during running could be explained by a greater applied force on the sandals. Indeed, the vertical ground reaction force measured in this experiment reached 2.5 to $2.9 \mathrm{BW}$ (body weight) for the passive and active peaks, respectively. This hypothesis could also work for the lack of difference during walking where the applied vertical force was about $1.2 \mathrm{BW}$.

The second objective of this study was to investigate the gait mode effect on foot restriction. The working hypothesis was that the gait mode affect the footwear constrain. Our results showed a diminution of the restriction due to the sandals during running relative to walking, thus confirming our hypothesis. This was supported by the DF excursion, $\Delta$ Bases, $\Delta$ Heads variables as well as the sole torsion amplitude. For all these variables, the foot restriction by sandals or straps was diminished during running compared to walking conditions. This could be explained regarding the amount of force acting on the sole for these two gait modes. This is likely supported by greater temporal coupling between forefoot, rearfoot and shank segments (Pohl et al. 2007) during running than during walking. DF excursion during walking was significantly smaller than during running while the first sole flexion peak increases from walking to running. Moreover, both EV and sole torsion amplitudes are greater during running trials. So the foot was less restrained by the sandals during running compared to walking as the speed of locomotion naturally enhanced the applied forces on the sandals.

One limitation of this study was the lack of difference in the foot motion between the both midsole hardness, 40C and 65C Asker. This was not due to a weakness in statistical tests because the observed powers of the ANOVAs were high (see Appendix A) and the Tukey test was a powerful post-hoc test for our purpose. As discussed by Kurz and Stergiou (2003), a similarity of the hardness between the experimental and the daily shoes could explain the subject adaptation and the lack of significance between conditions. So, either studies on specific hardness might be useless relative to the main shoe effect or future studies might look at more extreme midsole properties or designs. Another limitation was the strap tension of the sandals that was not measured, but controlled by the experimenter. Results might have been influenced by difference in strap tension, as Hagen and Hennig (2009) showed that the lacing method influenced the heel-toe running biomechanics.

The present findings highlighted few ideas of great interest. Firstly, in order to resemble natural foot motion and increase comfort during shod gait, normal forefoot spreading appeared to be important. Secondly, our results presented differences between walking and running conditions where the restriction seemed to diminish during running relative to walking. This finding showed that, combined with the increased speed of the different gait mode, the forces applied to govern the motion of the sole might be greater. Finally, clinicians need to be aware of the restrictions of forefoot motion that can be imposed by footwear. It can be assumed that too much restriction of the natural foot motion can be an important factor for the onset of foot pain and injury. It may be hypothesized that as soon as the normal width expansion of the forefoot during the loading phase of gait is prevented by footwear then local stress under the metatarsals may be elevated. According to this hypothesis, Hagen and Hennig (2009) looked at shoe-lacing effect on running biomechanics found an upper effect on plantar pressure distribution. These authors found a plantar pressure decrease under the lateral midfoot for higher and tighter shoe-lacing. Nevertheless, the influence of the constraint of the shoe upper on local plantar pressure needs further investigations especially concerning the forefoot. It is plausible that restriction of normal foot motion could be a contributing factor in the development of forefoot pathologies like stress fractures or foot deformities due to footwear (Kadambande et al., 2006; Zipfel and Berger, 2007). Therefore the shoe should provide a certain amount of flexibility that allows the forefoot to expand and perhaps limit internal stress at the foot-shoe interface.

\section{Conclusion}

This study focused on the sole motion as a parameter to discuss changes in forefoot to rearfoot relative motions. In summary, differences in foot motions between barefoot and shod conditions were induced by the sole and the forefoot spreading by the strap of the sandals. Foot motions were constrained by the footwear for forefoot to rearfoot EV/INV and ABD/ADD, but not for the PF/DF. Moreover, the sandal influenced the forefoot EV/INV motion during the push-off phase in shod conditions relative to barefoot. The influence of the midsole hardness on the sole torsion was also a relevant finding to gain further understanding about possible injury mechanisms and about the influence of shoe sole motions on foot motions. Further studies should look at the implication of the upper on the forefoot spreading and the influence of more extreme shoe designs and sole hardness conditions.

\section{Conflict of interest}

There is no conflict of interest to be reported for this manuscript.

\section{Acknowledgment}

The authors would like to thank the biomechanics staff of Liverpool John Moores University where the present study was conducted.

\section{Appendix A. Supporting Information}

Supplementary data associated with this article can be found in the online version at doi:10.1016/j.jbiomech.2009.06.015.

\section{References}

Carson, M.C., Harrington, M.E., Thompson, N., O'Connor, J.J., Theologis, T.N., 2001. Kinematic analysis of a multisegment foot model for research and clinical applications: a repeatability analysis. Journal of Biomechanics 34, 1299-1307. 
Eslami, M., Begon, M., Farahpour, N., Allard, P., 2007. Forefoot-rearfoot coupling patterns and tibial internal rotation during stance phase of barefoot versus shod running. Clinical Biomechanics 22, 74-80.

Freychat, P., Belli, A., Carret, J.P., Lacour, J.R., 1996. Relationship between rearfoot and forefoot orientation and ground reaction forces during running. Medicine and Science in Sports and Exercise 28, 225-232.

Hamill, J., Bates, B.T., Holt, K.G., 1992. Timing of lower extremity joint actions during treadmill running. Medicine and Science in Sports Exercise 24, 808-813.

Hardin, E.C., van den Bogert, A.J., Hamill, J., 2004. Kinematic adaptations during running: effects of footwear, surface, and duration. Medicine and Science in Sports and Exercise 36, 838-844.

Hagen, M., Hennig, E.M., 2009. Effects of different shoe-lacing patterns on the biomechanics of running shoes. Journal of Sports Sciences 27, 267-275.

Hunt, A.E., Smith, R.M., Torode, M., Keenan, A.M., 2001. Inter-segment foot motion and ground reaction forces over the stance phase of walking. Clinical Biomechanics 16, 592-600.

Kadambande, S., Khurana, A., Debnath, U., Bansal, M., Hariharan, K., 2006. Comparative anthropometric analysis of shod and unshod feet. The Foot 16, 188-191.

Kidder, S., Abuzzahad, F., Harris, G., Johnson, J., 1996. A system for the analysis of foot and ankle kinematics during gait. IEEE Transactions in Rehabilitation Engineering 4, 25-32.

Kurz, M.J., Stergiou, N., 2003. The spanning set indicates that variability during the stance period of running is affected by footwear. Gait \& Posture 17, 132-135.

Leardini, A., Benedetti, M.G., Catani, F., Simoncini, L., Giannini, S., 1999. An anatomically based protocol for the description of foot segment kinematics during gait. Clinical Biomechanics 14, 528-536.

Lee, S., Muller, C.C., Stefanyshyn, D., Nigg, B.M., 1999. Relative forefoot abduction and its relationship to foot length in vitro. Clinical Biomechanics 14,193-202.

Nigg, B.M., Bahlsen, H.A., 1988. Influence of heel flare and midsole construction on pronation, supination, and impact forces for heel-toe running. International Journal of Sport Biomechanics 4, 205-219.

Oleson, M., Adler, D., Goldsmith, P., 2005. A comparison of forefoot stiffness in running and running shoe bending stiffness. Journal of Biomechanics 38, 1886-1894.
Pohl, M.B., Buckley, J.G., 2008. Changes in foot and shank coupling due to alterations in foot strike pattern during running. Clinical Biomechanics 23, 334-341.

Pohl, M.B., Messenger, N., Buckley, J.G., 2006. Changes in foot and lower limb coupling due to systematic variations in step width. Clinical Biomechanics 21 , 175-183.

Pohl, M.B., Messenger, N., Buckley, J.G., 2007. Forefoot, rearfoot and shank coupling: effect of variations in speed and mode of gait. Gait \& Posture 25, 295-302.

Rattanaprasert, U., Smith, R., Sullivan, M., Gilleard, W., 1999. Three-dimensiona kinematics of the forefoot, rearfoot, and leg without the function of tibialis posterior in comparison with normals during stance phase of walking. Clinical Biomechanics 14, 14-23.

Schwanitz, S., Odenwald, S., 2008. Long-term cushioning properties of running shoe (P152). The Engineering of Sport 7, 95-100.

Stacoff, A., Kälin, X., Stüssi, E., Segesser, B., 1989. The torsion of the foot in running. International Journal of Sport Biomechanics 5, 375-389.

Stacoff, A., Kälin, X., Stüssi, E., 1991. The effect of shoes on the torsion and rearfoot motion in running. Medicine and Science in Sports and Exercise 23, 428-490.

Stacoff, A., Steger, J., Stüssi, E., Reinschmidt, C., 1996. Lateral stability in sideward cutting movements. Medicine and Science in Sports and Exercise 28, 350-358.

Stacoff, A., Nigg, B.M., Reinschmidt, C., van den Bogert, A.J., Lundberg, A., 2000. Tibiocalcaneal kinematics of barefoot versus shod running. Journal of Biomechanics 33, 1387-1395.

Stacoff, A., Reinschmidt, C., Nigg, B.M., van den Bogert, A.J., Lundberg, A., Denoth, J., Stüssi, E., 2001. Effect of shoe sole construction on skeletal motion during running. Medicine and Science in Sports and Exercise 33, 311-319.

Wu, G., Siegler, S., Allard, P., Kirtley, C., Leardini, A., Rosenbaum, D., Whittle, M. D'Lima, D.D., Cristofolini, L., Witte, H., Schmid, O., Stokes, I., 2002. ISB recommendation on definitions of joint coordinate system of various joints for the reporting of human joint motion-part I: ankle, hip, and spine. Journal of Biomechanics 35, 543-548.

Wolf, S., Simon, J., Patikas, D., Schuster, W., Armbrust, P., Döderlein, L., 2008. Foo motion in children shoes-a comparison of barefoot walking with shod walking in conventional and flexible shoes. Gait \& Posture 27, 51-59.

Zipfel, B., Berger, L.R., 2007. Shod versus unshod: the emergence of forefoot pathology in modern humans?. The Foot 17, 205-213. 